\title{
Naringenin reduces oxidative stress and improves mitochondrial dysfunction via activation of the Nrf2/ARE signaling pathway in neurons
}

\author{
KAIHUA WANG $^{1 *}$, ZHENZHEN CHEN $^{1,2^{*}}$, LONGJIAN HUANG ${ }^{1}$, \\ BING MENG ${ }^{1}$, XINMEI ZHOU ${ }^{1}$, XIAODONG WEN ${ }^{1}$ and DING REN ${ }^{1}$ \\ ${ }^{1}$ Department of Neurology, Ruikang Hospital Affiliated to Guangxi University of Chinese Medicine, Nanning, \\ Guangxi 530011; ${ }^{2}$ Guangxi University of Chinese Medicine, Nanning, Guangxi 530001, P.R. China
}

Received January 3, 2017; Accepted August 25, 2017

DOI: 10.3892/ijmm.2017.3134

\begin{abstract}
Oxidative stress and mitochondrial dysfunction are considered to be major contributing factors in the development and progression of many neurodegenerative diseases. Naringenin (NAR) is an abundant flavanone in the Citrus genus and has been found to exert antioxidant, anticarcinogenic and antimutagenic effects. However, the potential underlying mechanism of its antioxidant effects remains unclear. In the present study, the authors investigated the antioxidant effect of NAR on neurons in vitro. Neurons isolated from the brains of Sprague-Dawley rats were randomly divided into a control group, model group, NAR-L group, NAR-M group and NAR-H group. The model group received hypoxia and re-oxygenation treatment, and the NAR-L, NAR-M and NAR-H groups received 20, 40 and $80 \mu \mathrm{M}$ NAR, respectively. The levels of reactive oxygen species (ROS) in each group were detected by chloromethyl-2',7'dichlorodihydro fluorescein diacetate staining, and differences in mitochondrial
\end{abstract}

Correspondence to: Dr Ding Ren, Department of Neurology, Ruikang Hospital Affiliated to Guangxi University of Chinese Medicine, 10 Huadong Road, Nanning, Guangxi 530011, P.R. China E-mail: 360697490@qq.com

${ }^{*}$ Contributed equally

Abbreviations: NAR, naringenin; ROS, reactive oxygen species; GSH, glutathione; MDA, malondialdehyde; SOD1, superoxide dismutase 1; ANT, adenine nucleotide translocase; Nrf2, nuclear factor erythroid 2-related factor 2; ARE, antioxidant response element; Keap1, kelch-like ECH-associated protein 1; CM- $\mathrm{H}_{2}$ DCFDA, chloromethyl2',7'dichlorodihydro fluorescein diacetate; $\Delta \psi \mathrm{m}$, mitochondrial membrane potential; HPLC, high performance liquid chromatography; siRNA, small interfering RNA; siNfr2, anti-Nrf2 siRNA; RT-qPCR, reverse transcription-quantitative polymerase chain reaction; HO-1, heme oxygenase 1; NQO1, NAD(P)H quinone dehydrogenase 1; Bcl-2, B-cell lymphoma 2; Bax, Bcl-2-associated X protein

Key words: naringenin, oxidative stress, mitochondrial dysfunction, nuclear factor erythroid 2-related factor 2/antioxidant response element, neurons dysfunction were analyzed through measurement of mitochondrial membrane potential $(\Delta \psi \mathrm{m})$, adenine nucleotide translocase transport activity and adenine nucleotide levels. MTT and flow cytometry assays were also used to analyze cell proliferation and apoptosis, and the effects of NAR on the nuclear factor erythroid 2-related factor 2 (Nrf2)/antioxidant response element (ARE) signaling pathway were investigated using small interfering RNA methods. The authors detected an increased accumulation of ROS in the model group, and high-dose NAR could significantly reduce the levels of ROS. Furthermore, NAR could improve mitochondrial dysfunction, as indicated by increased levels of high-energy phosphates, enhanced mitochondrial ANT transport activity and increased mitochondrial membrane potential. Moreover, NAR increased cell viability and decreased the rate of cell apoptosis. NAR also increased the expression of Nrf2 and its downstream target genes. These findings demonstrated that NAR could reduce oxidative stress and improve mitochondrial dysfunction via activation of the Nrf2/ARE signaling pathway in neurons.

\section{Introduction}

Ischemic cerebrovascular disease, such as ischemic stroke, is a leading cause of death in developed countries and can severely impact affected individuals $(1,2)$. Ischemic stroke is primarily caused by deprivation of blood flow to regions of the brain, and results in deficiencies in oxygen and glucose supply (3). During ischemic stroke, mitochondrial dysfunction and calciumactivated proteolysis, accompanied by a complex cascade of damaging events including the generation of reactive oxygen species (ROS), ultimately led to the death of neurons (4-7). Therefore, targeting of the oxidant signaling pathway and antioxidant treatments may represent a novel therapeutic strategy for the treatment of ischemic cerebrovascular disease.

Nuclear factor erythroid 2-related factor 2 (Nrf2) is a basic leucine zipper protein and a redox-sensitive member of the cap'n'collar family of transcription factors $(8,9)$. It has been demonstrated in previous studies that Nrf2 and the antioxidant response element (ARE) pathway exerted protective effects against the generation of oxidative stress in mammalian neurons $(10,11)$. The activity of Nrf2 is negatively regulated 
by kelch-like ECH-associated protein 1 (Keap1). Under basal conditions, Nrf2 is primarily localized in the cytoplasm and is associated with Keap1 (12). This Keap1/Nrf2 complex is targeted for ubiquitination and proteasomal degradation, thereby maintaining quiescence of $\mathrm{Nrf} 2$ activity (13). In response to stress, Nrf2 is released from Keap1 via phosphorylation and translocates to the nucleus to carry out its transcriptional activities (14). Therefore, the discovery of new molecules that modulate the Nrf2/ARE pathway may aid in designing new strategies for the treatment of oxidative stress-related diseases.

The flavanone naringenin (NAR) is a major antioxidant present in citrus fruits, and has been found to exert antioxidant, anticarcinogenic and antimutagenic effects (15). Several studies have documented that NAR inhibits the growth of various types of cancer cells through the inhibition of cell proliferation and activation of apoptosis $(16,17)$. Regarding its antioxidant effect, research has indicated that NAR may reduce lactate dehydrogenase leakage and ROS generation, increase mitochondrial membrane potential, and reduce caspase-3/7 activity and DNA damage $(18,19)$. However, the effects of NAR on ischemic cerebrovascular disease, particularly ischemic stroke, remain unclear. In the present study, the authors demonstrated that NAR could reduce oxidative stress and improve the mitochondrial dysfunction in vitro via activation of the Nrf2/ARE signaling pathway in neurons.

\section{Materials and methods}

Cell culture and treatments. A total of 10 neonatal SpragueDawley (SD) rats (weighing, 5-6 g, uncertain gender, fed by breastfeeding, kept in an environment at $21 \pm 2^{\circ} \mathrm{C}$ ) were obtained from the Experimental Animal Center of Southern Medical University, (Guangzhou, China). The institutional review boards of all participating centers approved the protocols used in the study, and all experimental protocols were approved by the Review Committee for the Use of Human or Animal Subjects of Southern Medical University (Guangzhou, China; permit number: 2016213). Neurons were isolated through enzymatic digestion of the brain tissue of the rats, as previously described (20). Briefly, the rats were sacrificed by an overdose of $1 \%$ sodium pentobarbital ( $40 \mathrm{mg} / \mathrm{kg}$ per SD rat) administered via intraperitoneal injection, and then the cerebral cortices from neonatal SD rats (0-2 days old) were dissected and trypsinized with $0.125 \%$ trypsin for $30 \mathrm{~min}$ at $37^{\circ} \mathrm{C}$. Samples were passed through a $200-\mu \mathrm{m}$ mesh sieve and cells were collected by centrifugation $(500 \mathrm{x}$ g for $5 \mathrm{~min}$ ). Cells were resuspended in Dulbecco's modified Eagle's medium (DMEM) with 10\% fetal bovine serum (FBS; Gibco, Thermo Fisher Scientific, Inc., Waltham, MA, USA), $100 \mathrm{U} / \mathrm{ml}$ streptomycin and $100 \mathrm{U} / \mathrm{ml}$ penicillin, then seeded onto poly-D-lysine-coated $25-\mathrm{mm}$ dishes and cultured at $37^{\circ} \mathrm{C}$ in a $5 \% \mathrm{CO}_{2}$ atmosphere. Culture medium was replenished twice a week with fresh complete DMEM.

At $24 \mathrm{~h}$ after plating, neurons were divided into the following five groups: Control, model, NAR-L (low-dose), NAR-M (medium-dose) and NAR-H (high-dose). Neurons in the control group were cultured under normal conditions for 6 days. Neurons in the model group were cultured under normal conditions for 4 days, followed by oxygen deprivation $\left(90 \% \mathrm{~N}_{2}, 5 \% \mathrm{O}_{2}\right.$ and $5 \%$ $\mathrm{CO}_{2}$ ) for $12 \mathrm{~h}$ and oxygen restoration $\left(95 \%\right.$ air and $\left.5 \% \mathrm{CO}_{2}\right)$ for 36 h. In the NAR treatment groups, neurons were cultured under normal conditions for 4 days, then co-cultured with different concentrations of NAR $(20 \mu \mathrm{M}$ for NAR-L, $40 \mu \mathrm{M}$ for NAR-M and $80 \mu \mathrm{M}$ for NAR-H) for $8 \mathrm{~h}$, followed by oxygen deprivation for $12 \mathrm{~h}$ and oxygen restoration for $36 \mathrm{~h}$. In the time-course assay, neurons were co-cultured with $80 \mu \mathrm{M}$ NAR for $0,2,4$ and $8 \mathrm{~h}$ prior to oxygen deprivation/reoxygenation, and assessed by reverse transcription-quantitative polymerase chain reaction (RT-qPCR) and western blot analysis.

Measurement of ROS. The generation of ROS was measured by chloromethyl-2',7'dichlorodihydro fluorescein diacetate (CM- $\mathrm{H}_{2} \mathrm{DCFDA}$ ) staining. For CM- $\mathrm{H}_{2} \mathrm{DCFDA}$ staining, cells were washed with ice cold PBS and treated with $10 \mu \mathrm{M}$ CM- $\mathrm{H}_{2}$ DCFDA (Invitrogen, Thermo Fisher Scientific) in DMEM for $45 \mathrm{~min}$. Cells were then trypsinized and suspended in phosphate-buffered saline (PBS) and fluorescence was measured at $538 \mathrm{~nm}$. Furthermore, the activities of superoxide dismutase 1 (SOD1) and glutathione (GSH), and the content of malondialdehyde (MDA), in the different groups were determined to assess the anti-oxidative effects of NAR using commercial kits (Nanjing KeyGen Biotech Co., Ltd., Nanjing, China).

Determination of mitochondrial adenine nucleotide translocase (ANT) transport activity in neurons. The authors determined mitochondrial ANT transport activity using an inhibitor termination method. A total of $20 \mu \mathrm{l}^{3} \mathrm{H}$-ADP solution $(0.3 \mu \mathrm{mol} / \mathrm{l})$ was added into $50 \mu \mathrm{l}$ of prepared mitochondrial suspension for $10 \mathrm{sec}$, then $50 \mu \mathrm{l}$ of the ANT inhibitor atractyloside $(3.2 \mathrm{nmol} / \mathrm{l})$ was added and mixed immediately to terminate ANT transporter function. The mixture was centrifuged for $20 \mathrm{~min}$ at $3,000 \mathrm{x} \mathrm{g}$ and $4^{\circ} \mathrm{C}$ and the supernatant was discarded. After resuspension and washing with $1 \mathrm{ml}$ of separation medium, $400 \mu \mathrm{l} \mathrm{H}_{2} \mathrm{O}_{2}(8.8 \mathrm{~mol} / \mathrm{l})$ was added to the precipitate, which was subsequently incubated in a $70^{\circ} \mathrm{C}$ water bath for $40 \mathrm{~min}$. ANT transport activity was measured using the liquid scintillation counting method (Triathler liquid scintillation counter; Triathler, Turku, Finland). ANT transport activity is positive proportional to the number of scintillation counts $(\mathrm{dpm} / \mathrm{sec})$, and the number of scintillation counts can reflect the change in ANT transport activity of different groups.

Measurement of mitochondrial membrane potential $(\Delta \psi m)$. The authors used JC-1 staining to measure mitochondrial depolarization in neurons of the control, model, NAR-L, NAR-M and NAR-H groups. Following treatment, $200 \mu \mathrm{l}$ cells were collected and incubated for $20 \mathrm{~min}$ with an equal volume of JC-1 staining solution at $37^{\circ} \mathrm{C}$, then rinsed twice with PBS. $\Delta \psi \mathrm{m}$ was determined by measuring the relative amount of dual emissions from mitochondrial JC-1 monomers or aggregates using flow cytometry (BD Biosciences, Franklin Lakes, NJ, USA). $\Delta \psi \mathrm{m}$ was presented in units of fluorescence intensity.

Analysis of adenylate levels in neurons. The levels of adenine nucleotides in the neurons of each group were determined by high-performance liquid chromatography (HPLC), according to the method described by Yang et al (21). The following conditions were used for HPLC: wavelength, $254 \mathrm{~nm}$; sensitivity, 0.01 AUFS; mobile phase, $2 \mathrm{mmol} / 1 \mathrm{PBS}$ ( $\mathrm{pH} \mathrm{5.5);}$ and speed, $1 \mathrm{ml} / \mathrm{min}$. A YWG-ODS C18 $(10 \mu \mathrm{m})$ column $(4.6 \times 250 \mathrm{~nm})$ was used. 
Table I. The sequences of siRNAs used in the present study.

\begin{tabular}{lll}
\hline Gene & \multicolumn{1}{c}{ Name } & Sequence $\left(5^{\prime}-3^{\prime}\right)$ \\
\hline siNrf2 & siNrf2-sense & TGGAGCAAGTTTGGCAGGAGCTATT \\
& siNrf2-antisense & AATAGCTCCTGCCAAACTTGCTCCA \\
siRNA-NC & Control sense & GGAAAUCGAGUUCGCCGUU \\
& Control antisense & AACGGCGAACUCGAUUUCC \\
\hline
\end{tabular}

siRNA, small interfering RNA; Nrf2, nuclear factor erythroid 2-related factor 2; NC, negative control. The siRNA for Nrf2 and siRNA-NC were synthesized and purified by Shanghai GenePharma Co., Ltd. (Shanghai, China).

Cell transfection. Cells were harvested during the logarithmic growth phase. Small interfering RNAs (siRNAs) against Nrf2 (siNfr2) and negative control siRNAs (siRNA-NC) were purchased from Shanghai GenePharma Co., Ltd. (Shanghai, China) and transfected into cells using Lipofectamine 2000 (Invitrogen, Thermo Fisher Scientific), according to the manufacturer's protocol. The sequences of siNrf2 and siRNA-NC are presented in Table I. Following transfection, western blotting was used to assess the transfection efficiency, and model and NAR treatment groups were established prior to further experiments.

Measurement of cell proliferation. Cell viability was determined using an MTT assay. Briefly, neurons were plated onto 96 -well plates at a density of $1 \times 10^{4}$ cells/well. Following treatment, $20 \mu \mathrm{l} \mathrm{MTT}(5 \mathrm{mg} / \mathrm{ml})$ was added to each well and incubated for $4 \mathrm{~h}$. The medium was then removed and the formazan crystals were dissolved with dimethyl sulfoxide (DMSO). Absorbance was read at $570 \mathrm{~nm}$ with a microplate reader (Multiskan Spectrum; Thermo Fisher Scientific).

Flow cytometry. Apoptotic cells were recognized and distinguished from normal cells using an Annexin V-fluorescein isothiocyanate (FITC)/propidium iodide (PI) apoptosis kit for flow cytometric analysis, according to the manufacturer's instructions (Invitrogen, Thermo Fisher Scientific). After treatment, neurons were harvested and washed twice with cold PBS, then incubated with $5 \mu 1$ FITC-Annexin V and $5 \mu 1 \mathrm{PI}$ working solution $(100 \mu \mathrm{g} / \mathrm{ml})$ for $15 \mathrm{~min}$ in the dark at room temperature. Cellular fluorescence was measured by flow cytometric analysis ( BD Accuri C6; BD Biosciences) (22).

Western blotting. Neurons were lysed in a radioimmunoprecipitation assay buffer [50 mM Tris- $\mathrm{HCl}(\mathrm{pH} 7.4), 150 \mathrm{mM} \mathrm{NaCl}$, $1 \%$ NP-40] containing a protease inhibitor cocktail (Roche Diagnostics $\mathrm{GmbH}$, Basel, Switzerland) using standard procedures. The total proteins concentration was determined using a Bio-Rad protein assay system (Bio-Rad Laboratories, Inc., Hercules, CA, USA). A total of $50 \mu \mathrm{g}$ proteins were subjected to $12 \%$ SDS-PAGE, and then transferred onto nitrocellulose membranes (Millipore, Billerica, MA, USA). The membranes were blocked with $5 \%(\mathrm{w} / \mathrm{v})$ skimmed milk in $0.05 \%$ Trisbuffered saline with Tween-20 at room temperature for $2 \mathrm{~h}$. Immunoblotting was performed with primary antibodies against Nrf2 (ab31163, dilution 1:1,000; Abcam, Cambridge, MA, USA) and its downstream targets heme oxygenase 1 (HO-1, ab1324, dilution 1:250; Abcam) and NAD(P)H quinone
Table II. Primers used for reverse transcription-quantitative polymerase chain reaction analysis.

\begin{tabular}{ll}
\hline Gene & \multicolumn{1}{c}{ Sequence (5'-3') } \\
\hline Cytochrome $c$ & F: CAACAAGAACAAAGGTATCACC \\
& R: GGTGATACCTTTGTTCTTGTTG \\
Caspase-3 & F: CACTGGAATGTCAGCTCGCAATG \\
& R: CAGGTCCACAGGTCCGTTCGTTC \\
Bax & F: CAAGAAGCTGAGCGAGTGTCTCA \\
& R: GTCTGCAAACATGTCAGCTGCCAC \\
Bcl-2 & F: TGAAGTACATCCATTATAAGCTG \\
& R: CAGGTGGACCACAGGTGGCACA \\
Nrf2 & F: GCAACTCCAGAAGGAACAGG \\
RO-1 & R: AGGCATCTTGTTGGGAATG \\
& F: AAGATTGCGCAGAAGGCCATGG \\
NOO1 & F: GTCTTTGTGTTCCTCTGTCAGCAG \\
& R: CCAGTTGAGGTTCTAAGAC \\
& F: GTCCACACCCGCCACCAGTTC \\
& R: TCCCACCATCACACCCTGGTG
\end{tabular}

F, forward; R, reverse; Bax, Bcl-2-associated X protein; Bcl-2, B-cell lymphoma 2; Nrf2, nuclear factor erythroid 2-related factor 2; HO-1, heme oxygenase-1; NQO1, NAD(P)H quinone dehydrogenase 1. Primers were designed using Primer Express version 2.0 software. Primer specificity was confirmed using Primer-BLAST web software.

dehydrogenase 1 (NQO1, ab28947, dilution 1:1,000; Abcam). $\beta$-actin levels were used for normalization. The protein bands were scanned using ECL western blotting detection reagents (Pierce, Rockford, IL, USA) and quantified using a ChemiDoc image analysis system (Bio-Rad Laboratories).

RNA isolation and RT-qPCR analysis. Total RNA was extracted from the cultured neurons of each group using TRIzol reagent (Invitrogen, Thermo Fisher Scientific). A cDNA Synthesis kit (Takara Biotechnology Co., Ltd., Dalian, China) was used to synthesize cDNA according to the manufacturer's instructions. qPCR was performed using an iCycleriQ Detection System (Bio-Rad Laboratories) and interaction dye SYBR-Green. The sequences of the primers used are listed in Table II. GAPDH 
A
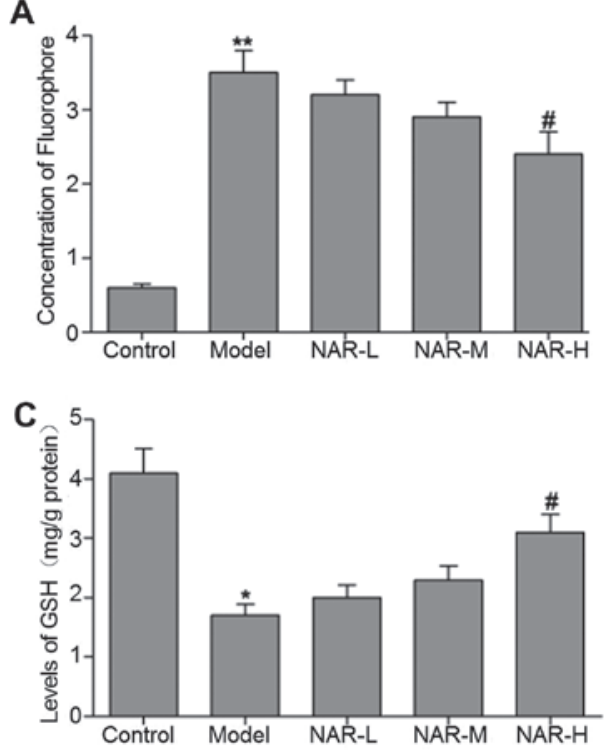

B

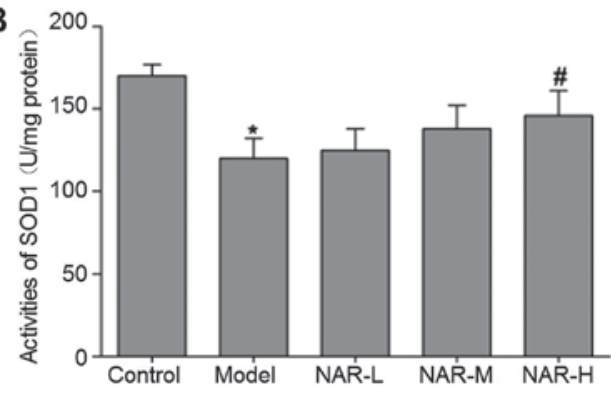

D

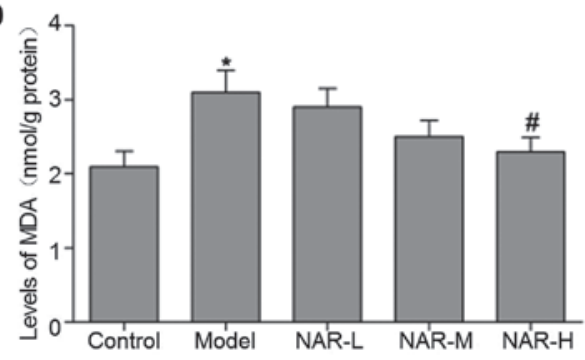

Figure 1. Effects of NAR on (A) reactive oxygen species accumulation, (B) SOD1 and (C) GSH activities and (D) MDA content. Data are presented as the mean \pm standard error of the mean $(n=3)$, and each bar represents the mean of three independent experiments carried out in triplicate. ${ }^{*} \mathrm{P}<0.05$, ${ }^{* *} \mathrm{P}<0.01 \mathrm{vs}$. control. ${ }^{\#} \mathrm{P}<0.05$ vs. model group. NAR, naringenin; SOD1, superoxide dismutase 1; GSH, glutathione; MDA, malondialdehyde.

mRNA levels were used for normalization. DNA was amplified with an initial denaturation at $95^{\circ} \mathrm{C}$ for $5 \mathrm{~min}$, followed by 39 cycles of $95^{\circ} \mathrm{C}(15 \mathrm{sec})$ and $60^{\circ} \mathrm{C}(15 \mathrm{sec})$. RT-qPCR data were analyzed and expressed as relative mRNA levels using CT values (23) and were subsequently converted to fold changes.

Statistical analysis. All the original experimental data were analyzed using the SPSS software (version 19.0; IBM SPSS, Armonk, NY, USA). Comparisons among the groups were performed by one-way analysis of variance (ANOVA), and Fisher's least significant difference test was used for comparisons between two groups. $\mathrm{P}<0.05$ was considered to indicate statistically significant differences.

\section{Results}

NAR reduces oxidative stress. To assess the antioxidant effect of NAR, ROS levels in the neurons of the different cell groups were analyzed by $\mathrm{CM}-\mathrm{H}_{2} \mathrm{DCFDA}$ staining. As presented in Fig. 1A, the authors observed an increased accumulation of ROS in the model group when compared with the control group $(\mathrm{P}<0.01)$. In turn, the levels of ROS were significantly decreased in the NAR-H group when compared with the model group $(\mathrm{P}<0.05)$. Additionally, compared with the model group, the high-dose NAR group exhibited significant increases in the activities of SOD1 and GSH $(\mathrm{P}<0.05$; Fig. 1B and $\mathrm{C})$. Furthermore, the level of MDA was significantly decreased in the NAR-H group when compared with the model group $(\mathrm{P}<0.05$; Fig. 1D). The levels of MDA and ROS, and activities of SOD1 and GSH, did not differ significantly between the NAR-L, NAR-M and model groups ( $\mathrm{P}>0.05)$.

NAR attenuates mitochondrial dysfunction. Mitochondria are considered to be a major producer of ROS in mammalian cells, and overproduction of ROS may be an indicator of mitochondrial dysfunction (24). Thus, the authors initially measured the
Table III. Effect of naringenin on the concentration of adenylate.

\begin{tabular}{llll}
\hline Group & $\begin{array}{c}\text { ATP } \\
(\mathrm{nmol} / \mathrm{mg})\end{array}$ & $\begin{array}{c}\text { ADP } \\
(\mathrm{nmol} / \mathrm{mg})\end{array}$ & $\begin{array}{c}\text { AMP } \\
(\mathrm{nmol} / \mathrm{mg})\end{array}$ \\
\hline Control & $2.84 \pm 0.21$ & $4.48 \pm 0.31$ & $4.88 \pm 0.38$ \\
Model & $1.11 \pm 0.11^{\mathrm{a}}$ & $1.74 \pm 0.15^{\mathrm{a}}$ & $3.53 \pm 0.28^{\mathrm{a}}$ \\
NAR-L & $1.26 \pm 0.11$ & $1.96 \pm 0.18$ & $3.61 \pm 0.29$ \\
NAR-M & $1.63 \pm 0.09$ & $2.36 \pm 0.16$ & $3.77 \pm 0.33$ \\
NAR-H & $2.46 \pm 0.19^{\mathrm{b}}$ & $3.93 \pm 0.34^{\mathrm{b}}$ & $4.37 \pm 0.35^{\mathrm{b}}$ \\
\hline
\end{tabular}

The adenine nucleotide in cardiomyocytes was determined using high-performance liquid chromatography method. Data are the mean \pm standard error of the mean. ${ }^{\mathrm{P}} \mathrm{P}<0.05$ vs. control group; ${ }^{\mathrm{b}} \mathrm{P}<0.05$ vs. model group.

concentration of adenylate in the different cell groups using a HPLC method. The data indicated that, compared with the control group, the levels of ATP, ADP and AMP decreased significantly in the model group, whereas the NAR-H groups exhibited notable improvements in adenylate levels when compared with the model group (Table III; $\mathrm{P}<0.05$ ).

ANT is a transporter of ATP and ADP in the mitochondrial membrane. ANT transport activity can reflect ATP synthesis and normal cell function. Therefore, the authors subsequently detected mitochondrial ANT transport activity in the neurons of the different cell groups. The data indicated that mitochondrial ANT transport activity was significantly decreased in the model group when compared with the control group $(\mathrm{P}<0.05)$, and significantly increased in NAR-H group when compared with the model group $(\mathrm{P}<0.05)$. However, ANT transport activity did not differ significantly between the 

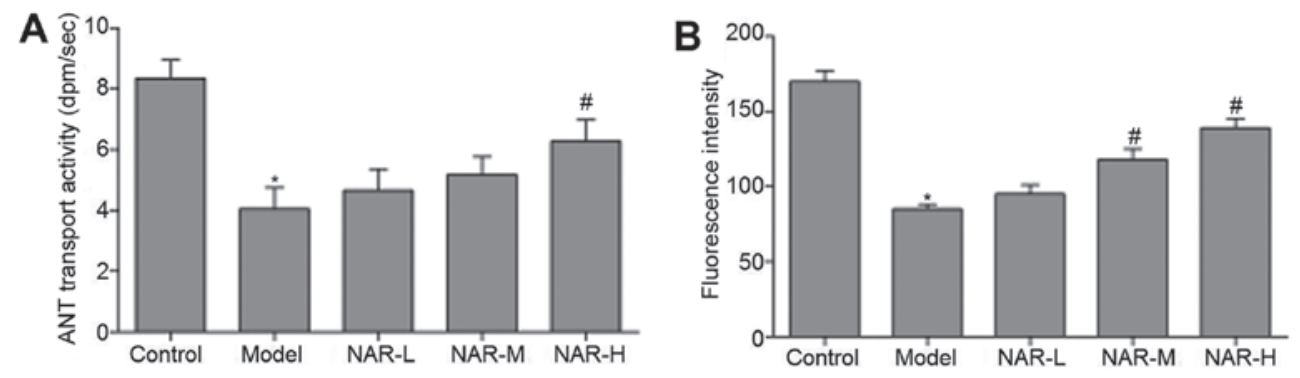

Figure 2. Effects of NAR on (A) mitochondrial ANT transport activity and (B) mitochondrial membrane potential. Data are presented as the mean \pm standard error of the mean $(\mathrm{n}=3)$, and each bar represents the mean of three independent experiments carried out in triplicate. " $\mathrm{P}<0.05$ vs. control. ${ }^{\#} \mathrm{P}<0.05$ vs. model group. NAR, naringenin; ANT, adenine nucleotide translocase.
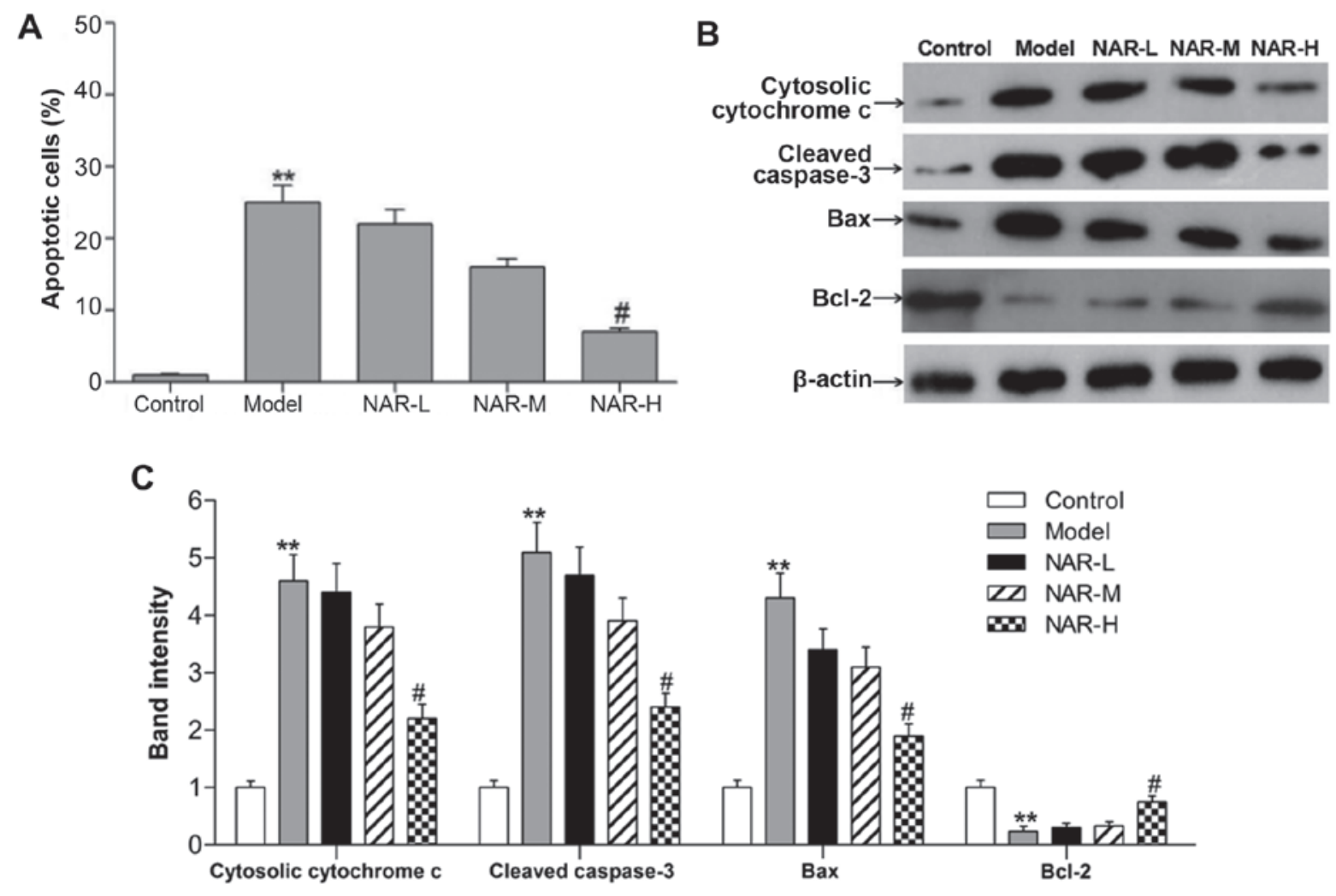

Figure 3. Effects of NAR on (A) apoptotic rate and (B and C) apoptotic marker expression. Cells were identified using an Annexin V-fluorescein isothiocyanate/propidium iodide apoptosis kit and flow cytometric analysis. Data are presented as the mean \pm standard error of the mean $(\mathrm{n}=3)$, and each bar represents the mean of three independent experiments carried out in triplicate. ${ }^{* *} \mathrm{P}<0.01$ vs. control. ${ }^{\text {}} \mathrm{P}<0.01$ vs. model group. NAR, naringenin; Bcl-2, B-cell lymphoma 2; Bax, Bcl-2-associated X protein.

NAR-L, NAR-M and model groups $(\mathrm{P}>0.05)$. These results are presented in Fig. 2A.

$\Delta \psi m$ was also analyzed by JC-1 staining. As in Fig. 2B, $\Delta \psi \mathrm{m}$ was significantly reduced in the model group when compared with the control group $(\mathrm{P}<0.05)$, while $\Delta \psi \mathrm{m}$ was markedly increased in the NAR-M and NAR-H groups when compared with the model group $(\mathrm{P}<0.05) . \Delta \psi \mathrm{m}$ did not differ significantly in the NAR-L group $(\mathrm{P}>0.05)$.

NAR increases cell viability and decreases cell apoptosis. It is well established that intracellular oxidative stress and mitochondrial dysfunction cause cell injury and neurotoxicity (25). Therefore, flow cytometry was performed to assess cell apoptosis in the different groups. As shown in Fig. 3A, the number of apoptotic cells was significantly increased in the model group when compared with the control group $(\mathrm{P}<0.01)$, while the number of apoptotic cells was significantly decreased in the NAR-H group when compared with the model group $(\mathrm{P}<0.05)$. To verify results of the apoptosis assay, western blot analysis was performed to detect markers of apoptosis, namely B-cell lymphoma 2 (Bcl-2), Bcl-2-associated X protein (Bax), cytosolic cytochrome $c$ and cleaved caspase-3 (Fig. 3B and C). A strong increase was observed in the expression of Bax and reduced expression of $\mathrm{Bcl}-2$ in the model group $(\mathrm{P}<0.01)$. Furthermore, the expression levels of cytosolic cytochrome $c$ and cleaved caspase-3 were markedly increased in the model group $(\mathrm{P}<0.01)$. However, high-dose NAR could reverse these expression changes $(\mathrm{P}<0.05)$. An MTT method was subsequently used to measure cell viability in the different groups. 


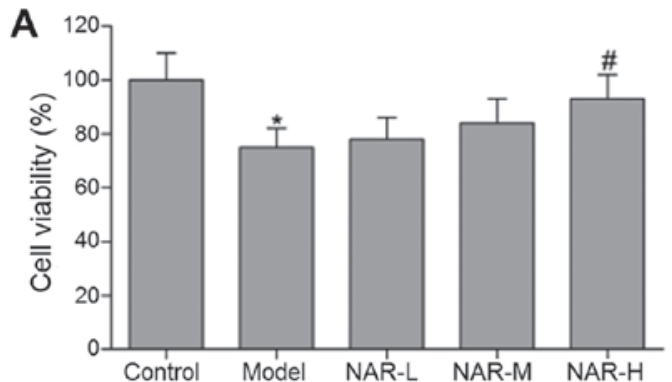

B
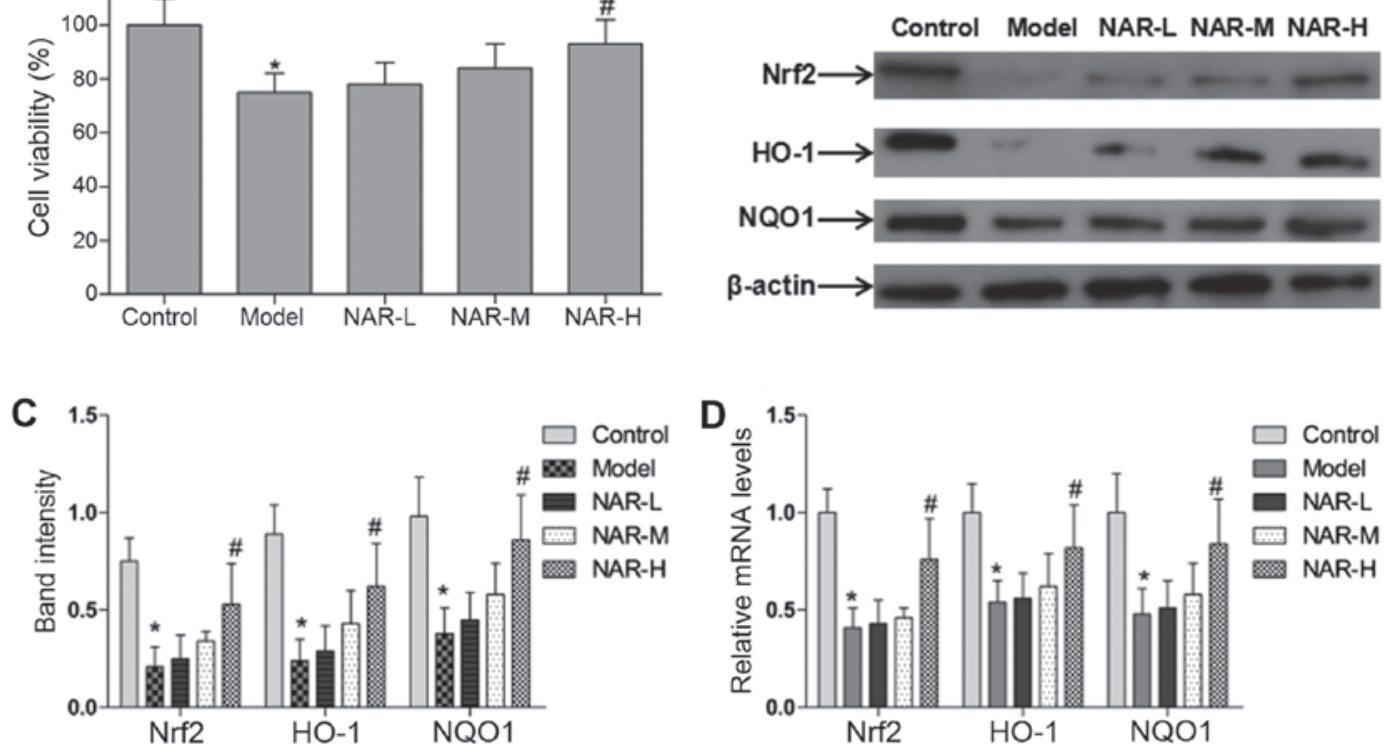

Figure 4. Effects of NAR on cell viability and Nrf2, HO-1 and NQO1 expression levels. (A) Cell viability was measured using an MTT assay. (B) Western blot images of Nrf2, HO-1 and NQO1 protein expression. (C) Statistical analysis of Nrf2, HO-1 and NQO1 protein levels. (D) Relative levels of Nrf2, HO-1 and NQO1 mRNA in each group. Data are presented as the mean \pm standard error of the mean $(n=3)$, and each bar represents the mean of three independent experiments carried out in triplicate. ${ }^{*} \mathrm{P}<0.05$ vs. control. ${ }^{~} \mathrm{P}<0.05$ vs. model group. NAR, naringenin; Nrf2, nuclear factor erythroid 2-related factor 2; HO-1, heme oxygenase-1; $\mathrm{NQO} 1, \mathrm{NAD}(\mathrm{P}) \mathrm{H}$ quinone dehydrogenase 1.
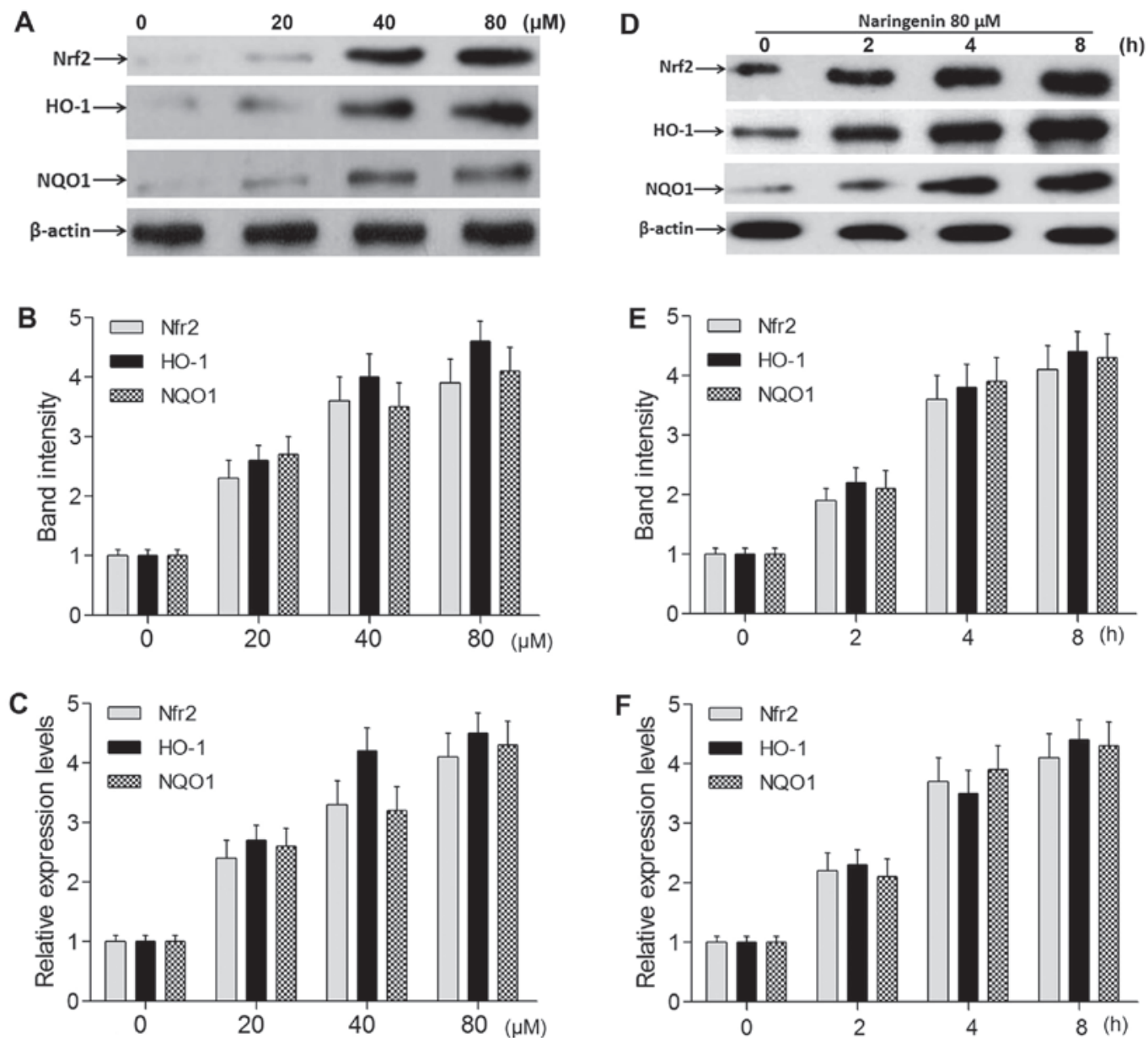

Figure 5. NAR induced the expression of Nrf2 and its target genes in an apparent concentration- and time-dependent manner. (A and D) Western blot images of Nrf2, HO-1 and NQO1 protein expression. (B and E) Statistical analysis of Nrf2, HO-1 and NQO1 protein levels. (C and F) Relative levels of Nrf2, HO-1 and NQO1 mRNA. Data are presented as the mean \pm standard error of the mean $(n=3)$, and each bar represents the mean of three independent experiments carried out in triplicate. NAR, naringenin; Nrf2, nuclear factor erythroid 2-related factor 2; HO-1, heme oxygenase-1; NQO1, NAD(P)H quinone dehydrogenase 1. 

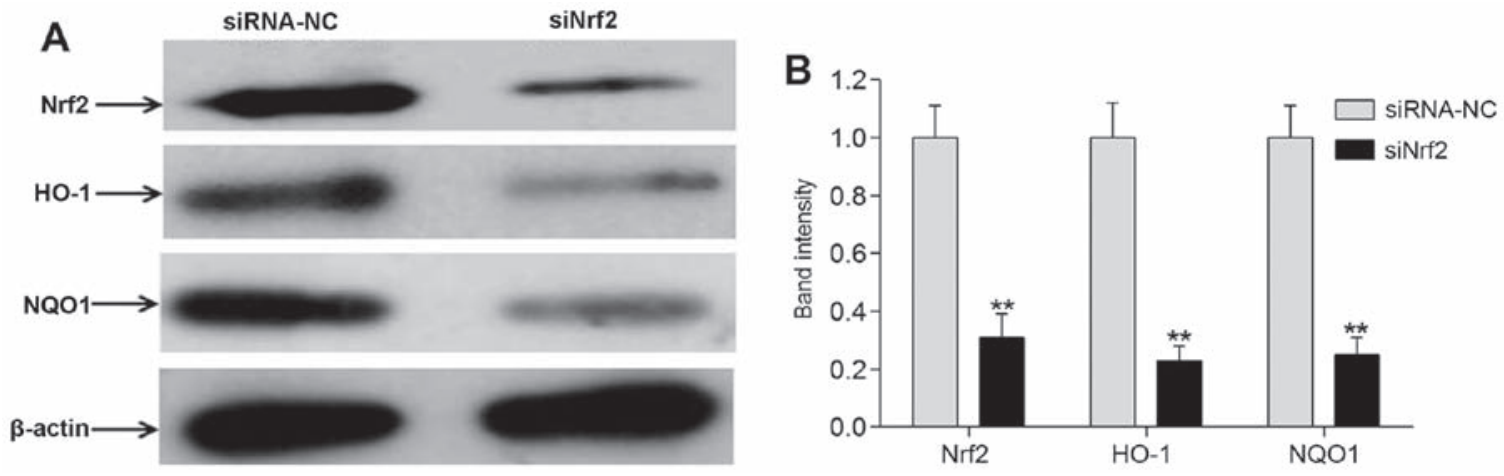

Figure 6. (A and B) Effects of NAR on the expression of Nrf2 and its target genes following transfection with siNrf2. Data are presented as the mean \pm standard error of the mean $(\mathrm{n}=3)$, and each bar represents the mean of three independent experiments carried out in triplicate. ${ }^{* *} \mathrm{P}<0.05$ vs. siRNA-NC group. NAR, naringenin; Nrf2, nuclear factor erythroid 2-related factor 2; si, small interfering; NC, negative control; HO-1, heme oxygenase-1; NQO1, NAD(P)H quinone dehydrogenase 1 .

A
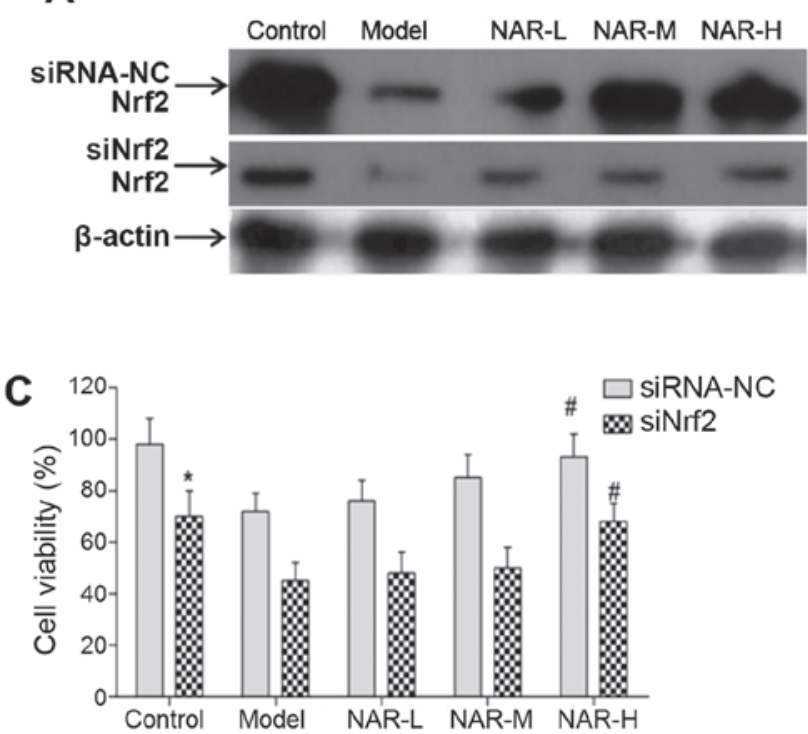
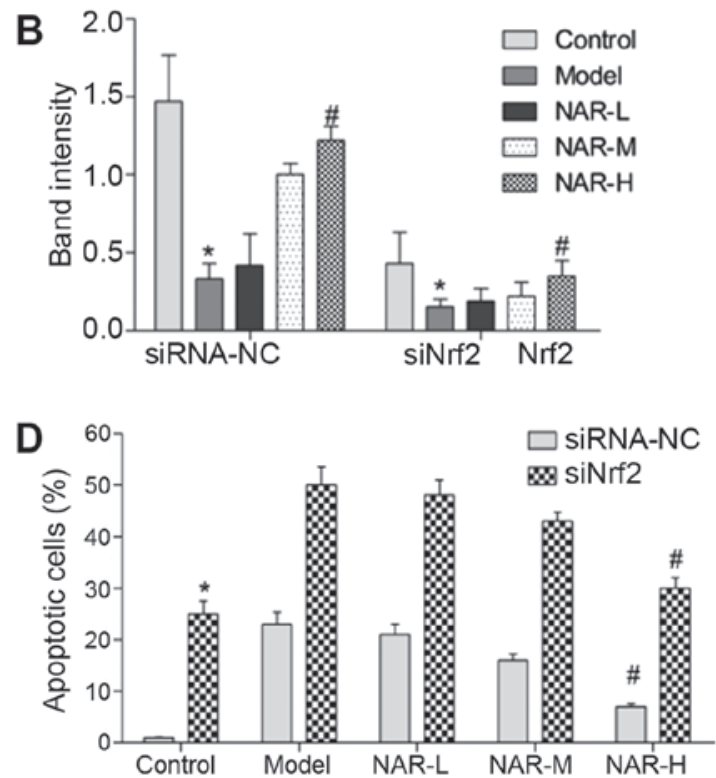

Figure 7. Effects of NAR on cell viability and apoptosis following transfection with siNrf2. (A) Western blot images of Nrf2 protein expression in the siRNANC and siNrf2 cells groups. (B) Statistical analysis of Nrf2 protein levels. (C) Cell viability was measured using an MTT assay. (D) The rate of apoptosis was measured by flow cytometry. Data are presented as the mean \pm standard error of the mean $(n=3)$, and each bar represents the mean of three independent experiments carried out in triplicate. ${ }^{*} \mathrm{P}<0.05$ vs. siRNA-NC group; ${ }^{*} \mathrm{P}<0.05$ vs. respective transfected model group. NAR, naringenin; Nrf2, nuclear factor erythroid 2-related factor 2; si, small interfering; NC, negative control.

The data indicated that the viability of cells was significantly decreased in the model group and increased in the NAR-H group $(\mathrm{P}<0.05$; Fig. 4A). However, neuronal viability, along with the rate and markers of apoptosis, did not differ significantly in the NAR-L and NAR-M groups ( $\mathrm{P}>0.05)$.

NAR increases the gene expression of Nrf2 and its target genes. The authors performed RT-qPCR and western blot analysis to measure the expression level of Nrf2 and its target genes in oxidative stressed neurons following treatment with different concentrations of NAR. The experiments demonstrated that the protein and mRNA levels of Nrf2 and its target genes HO-1 and NQO1 were significantly decreased in the model group and increased in the NAR-H group ( $\mathrm{P}<0.05$; Fig. 4B-D). In addition, NAR induced the transcription of Nrf2, HO-1 and NQO1 in an apparent dose- and time-dependent manner (Fig. 5).

To further explore the effect of NAR on neurons, Nrf2 was knocked down using RNA interference. Compared with the siRNA-NC cell group, the expression levels of Nrf2 and its target genes HO-1 and NQO1 were markedly reduced in the siNrf2 group, as demonstrated by western blot analysis $(\mathrm{P}<0.01$; Fig. 6). These data indicated that knockdown of Nrf2 using RNA interference was successful. Moreover, in both the siRNA-NC and siNrf2 cell groups, NAR-H treatment could increase the expression levels of Nrf2 when compared with transfected model neurons ( $\mathrm{P}<0.05$; Fig. 7).

In addition, the authors assessed the viability and apoptosis of neurons following Nrf2 knockdown. Compared with the siRNA-NC group, the viability of neurons was significantly decreased and the apoptosis of neurons was markedly increased in the siNrf2 cell group $(\mathrm{P}<0.05)$. These results indicated that Nrf2 could regulate the viability and apoptosis of neurons. Following NAR treatment, the data indicated that NAR could rescue the viability of neurons in the siRNA-NC and siNrf2 cells groups. Notably, NAR-H treatment significantly rescued 
the viability of transfected model neurons ( $\mathrm{P}<0.05$; Fig. 7C). In addition, the data indicated that NAR treatment could inhibit the apoptosis of neurons in the siRNA-NC and siNrf2 cell groups; NAR-H treatment significantly rescued the apoptosis of transfected model neurons ( $\mathrm{P}<0.05$; Fig. 7D).

Taken together, these data suggested that NAR could activate the Nrf2/ARE signaling pathway and regulate the gene expression of Nrf2 and its downstream targets.

\section{Discussion}

Evidence indicates that excessive formation of ROS and the resulting oxidative stress are key contributing factors in the aggravation of neuronal damage following ischemic insult (26). Under normal conditions, ROS serve roles in various crucial physiological processes, and can be rapidly scavenged by many endogenous antioxidant enzymes (27). During ischemia, ROS are over-produced to a level that exceeds the reductive capabilities of endogenous antioxidant systems, leading to neurotoxicity and, ultimately, neuronal cell death by necrosis and apoptosis $(28,29)$. Therefore, reducing oxidative stress could effectively increase cell viability and decrease cell apoptosis, and is a potential therapeutic strategy for the treatment of ischemic insult.

Mitochondria serve essential roles in energy production through the oxidative phosphorylation pathway, which supplies ATP for numerous intracellular biological processes $(30,31)$. Increasing evidence suggests that mitochondria are an important source of ROS, due to ROS leakage from the electron transport chain, while also being susceptible to oxidative damage, which leads to mitochondrial dysfunction $(32,33)$. In turn, mitochondrial dysfunction can result in mitochondrial fission, energy depletion and cell apoptosis $(34,35)$. Notably, mitochondrial dysfunction is considered to be a major contributing factor in the development and progression of many neurodegenerative conditions, such as Parkinson's, Huntington's and Alzheimer's disease, traumatic brain injury and ischemia-reperfusion injury $(36,37)$. Thus, decreasing mitochondrial dysfunction and improving mitochondrial function may be another promising target for the treatment of ischemic insult.

Flavonoids are major components of many edible plants and medicinal herbs, and have attracted a great deal of attention in previous years due to their reported antioxidative and antitumor effects in various chronic diseases $(38,39)$. Among them is NAR, an abundant flavanone in citrus fruits (40), and previous evidence has indicated that NAR offers protection against oxidative stress through its strong antioxidant activity (41). A previous study also reported that NAR reduced the necrosis of myocytes induced by ROS and exerted cytoprotective effects through its antioxidant activities (42). In the present study, the authors observed increased ROS accumulation in the model group and a lower ROS content in the NAR-H group. Moreover, high-dose NAR significantly increased the activities of SOD1 and GSH, and decreased the production of MDA. These data demonstrated that oxidative stress occurred during neuronal ischemic injury, and that a high concentration of NAR could effectively reduce this oxidative stress through the activation of endogenous antioxidant enzymes.

The authors further explored the effect of NAR on mitochondrial dysfunction. First, the levels of intracellular adenylate in each group were determined. The data indicated that ATP, ADP and AMP were significantly decreased in the model group, whereas adenylate levels in the NAR-H group were notably improved. In addition, ANT transport activity and $\Delta \psi \mathrm{m}$ were analyzed. The results indicated that ANT transport activity and $\Delta \psi \mathrm{m}$ were significantly reduced in the model group, but were markedly increased in the NAR-H group. These observations revealed that NAR could attenuate mitochondrial dysfunction by enhancing ANT transport activity and $\Delta \psi \mathrm{m}$ and restoring energy production.

It is well established that mitochondrial dysfunction can lead to cell death in various cell types (43). The viability and apoptosis of cells in each group were further investigated, and detected a decrease in viability and increase in apoptosis in cells of the model group. By contrast, cells in the NAR-H group exhibited increased viability and decreased apoptosis. Taken together, these results indicated that NAR can effectively reduce oxidative stress and ameliorate mitochondrial dysfunction to ultimately attenuate cell death.

Therapies targeting the Nrf2 pathway have previously become a promising strategy for the treatment of stroke (44). Nrf2 is a major regulator of several cytoprotective factors, including antioxidative enzymes and anti-inflammatory factors $(45,46)$. Activation of Nrf2 serves a pivotal role in enhancing the endogenous defense mechanisms that protect the brain against progressive ischemic damage and promote its recovery after stroke $(47,48)$. Moreover, activation of Nrf2 can upregulate the expression of several antioxidative enzymes, including HO-1 and NQO1. The present study verified that NAR-H treatment enhanced the relative gene expression of $\mathrm{Nrf} 2$ and its targets genes. In addition, NAR-H treatment could significantly rescue the viability of neurons following siRNA knockdown of Nrf2.

In conclusion, the results demonstrated that administration of NAR prior to oxygen deprivation/reoxygenation reduced oxidative stress and improved mitochondrial dysfunction via activation of the Nrf2/ARE signaling pathway in neurons. In future studies, in order to simulate the clinical status of ischemic cerebrovascular disease as much as possible, neurons could be exposed to hypoxia and then re-supplied with oxygen and NAR for different periods of time. After re-supplying oxygen and NAR, the cell viability, the levels of ROS generation, Nrf2 and its downstream genes and mitochondrial functions should be assayed.

\section{Acknowledgements}

The present study was supported by a class general financial grant from the Guangxi Natural Science Foundation (grant no. 2012GXNSFAA053076); the Youth Fund Project of the Guangxi Natural Science Foundation (grant nos. 2012GXNSFBA053082 and 2013GXNSFBA019153); and the Guangxi Scientific and Technological Research Projects of Universities (grant no. KY2015ZD062).

\section{References}

1. Zhang L, Zhang X, Zhang C, Bai X, Zhang J, Zhao X, Chen L, Wang L, Zhu C, Cui L, et al: Nobiletin promotes antioxidant and anti-inflammatory responses and elicits protection against ischemic stroke in vivo. Brain Res 1636: 130-141, 2016.

2. Gopinath K and Sudhandiran G: Naringin modulates oxidative stress and inflammation in 3-nitropropionic acid-induced neurodegeneration through the activation of nuclear factor-erythroid 2-related factor-2 signalling pathway. Neuroscience 227: 134-143, 2012. 
3. Sahota P and Savitz SI: Investigational therapies for ischemic stroke: Neuroprotection and neurorecovery. Neurotherapeutics 8: 434-451, 2011.

4. Albarracin SL, Stab B, Casas Z, Sutachan JJ, Samudio I, Gonzalez J, Gonzalo L, Capani F, Morales L and Barreto GE: Effects of natural antioxidants in neurodegenerative disease. Nutr Neurosci 15: 1-9, 2012.

5. Abdul-Muneer PM, Chandra $\mathrm{N}$ and Haorah J: Interactions of oxidative stress and neurovascular inflammation in the pathogenesis of traumatic brain injury. Mol Neurobiol 51: 966-979, 2015

6. Antoniou X, Falconi M, Di Marino D and Borsello T: JNK3 as a therapeutic target for neurodegenerative diseases. J Alzheimers Dis 24: 633-642, 2011.

7. Ashrafi G and Schwarz TL: The pathways of mitophagy for quality control and clearance of mitochondria. Cell Death Differ 20: 31-42, 2013.

8. Alfieri A, Srivastava S, Siow RC, Cash D, Modo M, Duchen MR, Fraser PA, Williams SC and Mann GE: Sulforaphane preconditioning of the Nrf2/HO-1 defense pathway protects the cerebral vasculature against blood-brain barrier disruption and neurological deficits in stroke. Free Radic Biol Med 65: 1012-1022, 2013

9. Chen PC, Vargas MR, Pani AK, Smeyne RJ, Johnson DA, Kan YW and Johnson JA: Nrf2-mediated neuroprotection in the MPTP mouse model of Parkinson's disease: Critical role for the astrocyte. Proc Natl Acad Sci USA 106: 2933-2938, 2009.

10. Cuadrado A, Moreno-Murciano P and Pedraza-Chaverri J: The transcription factor Nrf2 as a new therapeutic target in Parkinson's disease. Expert Opin Ther Targets 13: 319-329, 2009.

11. de Vries HE, Witte M, Hondius D, Rozemuller AJ, Drukarch B, Hoozemans J and van Horssen J: Nrf2-induced antioxidant protection: A promising target to counteract ROS-mediated damage in neurodegenerative disease? Free Radic Biol Med 45: 1375-1383, 2008

12. Du Y, Villeneuve NF, Wang XJ, Sun Z, Chen W, Li J, Lou H, Wong PK and Zhang DD: Oridonin confers protection against arsenic-induced toxicity through activation of the Nrf2-mediated defensive response. Environ Health Perspect 116: 1154-1161, 2008.

13. Jazwa A, Rojo AI, Innamorato NG, Hesse M, Fernández-Ruiz J and Cuadrado A: Pharmacological targeting of the transcription factor Nrf2 at the basal ganglia provides disease modifying therapy for experimental parkinsonism. Antioxid Redox Signal 14: 2347-2360, 2011

14. Kaidery NA, Banerjee R, Yang L, Smirnova NA, Hushpulian DM, Liby KT, Williams CR, Yamamoto M, Kensler TW, Ratan RR, et al: Targeting Nrf2-mediated gene transcription by extremely potent synthetic triterpenoids attenuate dopaminergic neurotoxicity in the MPTP mouse model of Parkinson's disease. Antioxid Redox Signal 18: 139-157, 2013.

15. Chen D, Chen MS, Cui QC, Yang H and Dou QP: Structureproteasome-inhibitory activity relationships of dietary flavonoids in human cancer cells. Front Biosci 12: 1935-1945, 2007.

16. Kawaii S, Tomono Y, Katase E, Ogawa K and Yano M: HL-60 differentiating activity and flavonoid content of the readily extractable fraction prepared from citrus juices. J Agric Food Chem 47: 128-135, 1999.

17. Kanno S, Tomizawa A, Ohtake T, Koiwai K, Ujibe $\mathrm{M}$ and Ishikawa M: Naringenin-induced apoptosis via activation of NF-kappaB and necrosis involving the loss of ATP in human promyeloleukemia HL-60 cells. Toxicol Lett 166: 131-139, 2006.

18. Youdim KA, Qaiser MZ, Begley DJ, Rice-Evans CA and Abbott NJ: Flavonoid permeability across an in situ model of the blood-brain barrier. Free Radic Biol Med 36: 592-604, 2004

19. Prasain JK, Carlson SH and Wyss JM: Flavonoids and agerelated disease: Risk, benefits and critical windows. Maturitas 66: 163-171, 2010

20. Hu ZL, Huang C, Fu H, Jin Y, Wu WN, Xiong QJ, Xie N, Long LH, Chen JG and Wang F: Disruption of PICK1 attenuates the function of ASICs and PKC regulation of ASICs. Am J Physiol Cell Physiol 299: C1355-C1362, 2010.

21. Yang Y, Zhang H, Li X, Yang T and Jiang Q: Effects of PPAR $\alpha /$ PGC-1 $\alpha$ on the myocardial energy metabolism during heart failure in the doxorubicin induced dilated cardiomyopathy in mice. Int J Clin Exp Med 7: 2435-2442, 2014.

22. Schmid I, Uittenbogaart CH and Giorgi JV: Sensitive method for measuring apoptosis and cell surface phenotype in human thymocytes by flow cytometry. Cytometry 15: 12-20, 1994.

23. Livak KJ and Schmittgen TD: Analysis of relative gene expression data using real-time quantitative PCR and the 2(-Delta Delta C(T)) Method. Methods 25: 402-408, 2001.
24. Chaturvedi RK and Flint Beal M: Mitochondrial diseases of the brain. Free Radic Biol Med 63: 1-29, 2013

25. Ciccone S, Maiani E, Bellusci G, Diederich M and Gonfloni S Parkinson's disease: A complex interplay of mitochondrial DNA alterations and oxidative stress. Int J Mol Sci 14: 2388-2409, 2013.

26. Cookson MR: Parkinsonism due to mutations in PINK1, parkin, and DJ-1 and oxidative stress and mitochondrial pathways. Cold Spring Harb Perspect Med 2: a009415, 2012.

27. Boveris A, Oshino N and Chance B: The cellular production of hydrogen peroxide. Biochem J 128: 617-630, 1972.

28. Dhalla NS, Temsah RM and Netticadan T: Role of oxidative stress in cardiovascular diseases. J Hypertens 18: 655-673, 2000.

29. Abdelmegeed MA and Song BJ: Functional roles of protein nitration in acute and chronic liver diseases. Oxid Med Cell Longev 2014: 149627, 2014.

30. Dai Z, Wu Z, Yang Y, Wang J, Satterfield MC, Meininger CJ, Bazer FW and Wu G: Nitric oxide and energy metabolism in mammals. Biofactors 39: 383-391, 2013

31. Dashdorj A, Jyothi KR, Lim S, Jo A, Nguyen MN, Ha J, Yoon KS, Kim HJ, Park JH, Murphy MP, et al: Mitochondria-targeted antioxidant MitoQ ameliorates experimental mouse colitis by suppressing NLRP3 inflammasome-mediated inflammatory cytokines. BMC Med 11: 178, 2013.

32. Wey MC, Fernandez E, Martinez PA, Sullivan P, Goldstein DS and Strong R: Neurodegeneration and motor dysfunction in mice lacking cytosolic and mitochondrial aldehyde dehydrogenases: Implications for Parkinson's disease. PLoS One 7: e31522, 2012.

33. Winklhofer KF: Parkin and mitochondrial quality control: Toward assembling the puzzle. Trends Cell Biol 24: 332-341, 2014.

34. Winterbourn CC: Reconciling the chemistry and biology of reactive oxygen species. Nat Chem Biol 4: 278-286, 2008.

35. Wu W, Xu H, Wang Z, Mao Y, Yuan L, Luo W, Cui Z, Cui T, Wang XL and Shen YH: PINK1-Parkin-mediated mitophagy protects mitochondrial integrity and prevents metabolic stressinduced endothelial injury. PLoS One 10: e0132499, 2015.

36. Zhang J and Ney PA: Reticulocyte mitophagy: Monitoring mitochondrial clearance in a mammalian model. Autophagy 6: 405-408, 2010.

37. Xu K, Puchowicz MA, Sun X and LaManna JC: Mitochondrial dysfunction in aging rat brain following transient global ischemia. Adv Exp Med Biol 614: 379-386, 2008.

38. Purushotham A, Tian M and Belury MA: The citrus fruit flavonoid naringenin suppresses hepatic glucose production from Fao hepatoma cells. Mol Nutr Food Res 53: 300-307, 2009.

39. Sohn E, Kim J, Kim CS, Kim YS, Jang DS and Kim JS: Extract of the aerial parts of Aster koraiensis reduced development of diabetic nephropathy via anti-apoptosis of podocytes in streptozotocin-induced diabetic rats. Biochem Biophys Res Commun 391: 733-738, 2010.

40. Rajadurai M, Prince PS and Prince M: Preventive effect of naringin on isoproterenol-induced cardiotoxicity in Wistar rats: An in vivo and in vitro study. Toxicology 232: 216-225, 2007.

41. Liu L, Xu DM and Cheng YY: Distinct effects of naringenin and hesperetin on nitric oxide production from endothelial cells. J Agric Food Chem 56: 824-829, 2008.

42. Pérez-Jiménez J, Neveu V, Vos F and Scalbert A: Identification of the 100 richest dietary sources of polyphenols: An application of the Phenol-Explorer database. Eur J Clin Nutr 64 (Suppl 3): S112-S120, 2010

43. Green DR and Kroemer G: The pathophysiology of mitochondrial cell death. Science 305: 626-629, 2004.

44. Kroemer G and Reed JC: Mitochondrial control of cell death. Nat Med 6: 513-519, 2000.

45. Wu J, Li Q, Wang X, Yu S, Li L, Wu X, Chen Y, Zhao J and Zhao Y: Neuroprotection by curcumin in ischemic brain injury involves the Akt/Nrf2 pathway. PLoS One 8: e59843, 2013.

46. Ren J, Fan C, Chen N, Huang J and Yang Q: Resveratrol pretreatment attenuates cerebral ischemic injury by upregulating expression of transcription factor $\mathrm{Nrf} 2$ and $\mathrm{HO}-1$ in rats. Neurochem Res 36: 2352-2362, 2011.

47. Fujita K, Maeda D, Xiao Q and Srinivasula SM: Nrf2-mediated induction of p62 controls Toll-like receptor-4-driven aggresomelike induced structure formation and autophagic degradation. Proc Natl Acad Sci USA 108: 1427-1432, 2011.

48. Wang B, Cao W, Biswal S and Doré S: Carbon monoxideactivated Nrf2 pathway leads to protection against permanent focal cerebral ischemia. Stroke 42: 2605-2610, 2011. 